\title{
Antropometría del Yunque y Articulaciones Incudo-maleolar e Incudo-estapedial en Humanos: Un Estudio Anatómico Directo
}

\author{
Anthropometry of the Incus and Incudomalleolar and Incudostapedial \\ Joints in Humans: A Direct Anatomical Study
}

Luis Miguel Ramirez* \& Luis Ernesto Ballesteros**

\begin{abstract}
RAMIREZ, L. M. \& BALLESTEROS, L. E. Antropometría del yunque y articulaciones incudo-maleolar e incudo-estapedial en humanos: Un estudio anatómico directo. Int. J. Morphol., 31(2):539-545, 2013.

RESUMEN: Los estudios actuales sobre variaciones anatómicas del yunque en humanos a partir del registro morfométrico son insuficientes. Las características estructurales y dimensionales del yunque son registradas en 23 muestras de población mestiza adulta Colombiana. Las medidas registradas involucraron el cuerpo, la articulación incudo-maleolar, el proceso largo, el proceso corto y la articulación incudo-estapedial. Solo ocho de los treinta y cinco registros tomados en este estudio pudieron ser comparados concienzudamente con otros estudios anteriores que mostraron similitudes. El largo total del yunque fue de 6,51 mm (DE 0,32), la longitud del proceso largo del yunque fue de 5,35 mm (DE 0,25), la distancia entre proceso largo y corta fue de $6,06 \mathrm{~mm}$ (DE 0,35) y la proporción de longitudes entre ambos procesos fue de 82,18\% (DE 8,9). Conforme a la población estudiada se encontró variación pareada en la anatomía del yunque en solo tres de los registros tomados, sin embargo, consideramos que es un hueso morfométricamente uniforme y estable.
\end{abstract}

PALABRAS CLAVE: Oscículos; Yunque; Articulación incudo-maleolar; Proceso largo; Proceso corto; Articulación incudo-estapedial.

\section{INTRODUCCIÓN}

El martillo, yunque y parcialmente el estribo sufren osificación endocondral originada de la zona mas posterior del primer arco branquial (cartílago de Meckel) (Nishizaki \& Anniko, 1997; Whyte et al., 2002; Whyte et al., 2003). El segundo arco branquial (cartílago de Reichert) se encarga de complementar el estribo.

Los tres oscículos se articulan sinovialmente y en la zona media articular, el yunque se encarga de unirlos a través de dos áreas articulares: las articulaciones incudo-maleolar e incudo-estapedial. La presencia de los oscículos es la de comunicar mecánicamente medios densos diferentes con comportamientos físicos disparejos; permitiendo que la energía sonora cruce de un estado aéreo a uno liquido sin perder potencia. Si la cadena oscicular no estuviera presente más del 90\% de la energía sonora no se transmitiría. (Olsewski, 1989; Olsewski, 1990).

La forma del yunque se parece más a un diente premolar achatado con dos raíces divergentes que son los procesos superior o corto (unida a la fosa incudica de la pared timpánica posterior), y el proceso inferior o largo que se encuentra paralela y medial al eje del manubrio del martillo y se une en ángulo recto al estribo. El martillo y el yunque rotan juntos alrededor de un eje postero-anterior que va desde el proceso corto y el ligamento posterior del yunque al ligamento anterior del martillo, así cuando la membrana y el manubrio del martillo se desplazan en dirección medial, el proceso largo del yunque se mueve en la misma dirección empujando la base del estribo (ubicado como un pistón y en ángulo recto) hacia el laberinto y la perilinfa. El yunque tiene dos carillas, una anterior para el martillo en forma de silla de montar y una inferior o lenticular para el estribo. La carilla articular de este hueso se ubica en una meseta que corona la parte superior del cuerpo y contiene dos vertientes divididas por una depresión media que acopla con la zona media y prominente de la carilla articular del martillo que reproduce la morfología de la carilla del yunque en negativo, formando juntos la articulación incudo-maleolar (Guyton \& Hall, 2007). En comparación con la carilla incudo-estapedial del mismo hueso, es la más grande de las dos. Desde la perspectiva morfológica y morfométrica se debe matizar el hecho de que estas estructuras óseas son irregulares e imbalanceadas, lo que genera efecto de palanca mecánica en la transmisión sonora debido a los momentos de fuerza que esta morfología genera (Ramirez \& Ballesteros, 2010).

\footnotetext{
* Profesor Asociado. Facultad de Odontología, Grupo Estomatología Biomédica, Universidad de Antioquia, Medellín, Colombia.

** Profesor Titular. Grupo Variaciones Anatómicas y Biomecánica Tendomuscular, Universidad Industrial de Santander, Colombia.
} 
Se reportaron los primeras medidas de huesecillos del oído medio en el medioevo y aunque la base de datos recientemente a aumentado, no es mucha la información en relación a la morfometría de estos (Cole, 1949). Parece haber un interés común en la morfología y morfometría de la cadena de oscículos del oído medio en humanos con fines relacionados a la evolución, igualmente quirúrgicos pero también en el diseño y la reconstrucción de implantes electromagnéticos, usando medidas precisas que logran reproducir la transmisión de la energía sonora. (Mills, 1991; Nishihara \& Goode, 1994; aWengen et al., 1995; Asai et al., 1999; Whyte et al., 2003; Murugasu et al., 2005; Farahani \& Nooranipour, 2008).

\section{MATERIAL Y MÉTODO}

Se disecó el yunque mediante micro-disección de bloques de hueso temporal obtenidos de especímenes cadavéricos adultos frescos de raza mestiza, a quienes se les practicó autopsia en el Instituto de Medicina Legal de Bucaramanga (Colombia) con un abordaje superior a través de la fosa craneal media. El receso epitimpánico fue removido con motor y expuesta la cavidad ótica media en donde se luxó el yunque en la articulación incudo-maleolar e incudo-estapedial y posteriormente se desinserto de su ligamento posterior. Se limpiaron con agua oxigenada durante 2 horas y registraron morfométricamente. La toma de medidas en las muestras de yunque las realizo un solo operador y de manera directa en cada hueso, con calibrador electrónico (Mitutoyo®) y microscopia de aumento de 12x. Los registros fotográficos fueron logrados con video-microscopio de 50x.

Las zonas medidas en el cuerpo y procesos del yunque (Tabla I-V y Figs. 1-5) corresponden a las longitudes de sus extremos, al ancho y grosor en diferentes zonas y al ancho medio-lateral de la meseta y carilla articular en sus porciones posterior, medio y anterior, así como la longitud postero-anterior de la carilla.

Se realizaron medidas paramétricas (promedio y desviación estándar) de cada variable y una prueba T pareada en 22 de los especímenes que correspondieron a muestras izquierdas y derechas de un mismo individuo.

\section{RESULTADOS}

En las Tablas I, II, III, IV y V se encuentran las treinta y cinco medidas pormenorizadas para cada registro de los veintitrés especímenes de yunque estudiadas, con sus promedios y dispersiones individuales. Correspondientes con las tablas, las figuras 1, 2, 3, 4 y 5 muestran los sitios de cada registro.

Tabla I. Medidas del yunque en cuerpo, proceso corto y largo (Ver Fig. 1).

\begin{tabular}{lccccccc}
\hline Medida & $\begin{array}{c}\text { Proc } \\
\text { Largo-Ful }\end{array}$ & $\begin{array}{c}\text { Proc } \\
\text { Corto-Ful }\end{array}$ & Largo-Corto & Ful-Car P & $\begin{array}{c}\text { Ful-Car } \\
\mathbf{1} / 2-\mathbf{R}\end{array}$ & $\begin{array}{c}\text { Ful-Car } \\
\mathbf{1 / 2}-\mathbf{C}\end{array}$ & Ful-Car A \\
\hline X & 3,18 & 2,83 & 6,06 & 3,60 & 2,13 & 1,49 & 2,51 \\
DE & 0,24 & 0,19 & 0,35 & 0,22 & 0,17 & 0,16 & 0,16 \\
Rango & $2,69-3,56$ & $2,27-3,19$ & $5,32-6,55$ & $3,13-4,01$ & $1,85-2,48$ & $1,09-1,74$ & $2,18-2,74$ \\
\hline
\end{tabular}

Proc Largo-Ful: Longitud ápice de proceso corto a fulcro de los dos procesos (1 en Fig. 1).

Proc Corto-Ful: Longitud ápice de proceso largo a fulcro de los dos procesos (2 en Fig. 1).

Proc-Corto: Longitud entre ápices de proceso largo y proceso corto (3 en Fig. 1).

Ful-Car P: Longitud desde fulcro a zona más rostral y posterior de carilla articular incudo-maleolar (4 en Fig. 1).

Ful-Car 1/2-R: Longitud desde fulcro a zona media y más rostral (lateral) de carilla articular incudo-maleolar (5 en Fig. 1).

Ful-Car 1/2-C: Longitud desde fulcro a zona media y más caudal (medial) de carilla articular incudo-maleolar (6 en Fig. 1).

Ful-Car A: Longitud desde fulcro a zona más caudal y anterior de carilla articular incudo-maleolar (7 en Fig. 1).

Tabla II. Medidas del grosor y ancho proximal y apical de procesos corto y largo del yunque (Ver Fig. 2).

\begin{tabular}{llllllll}
\hline Medida & $\begin{array}{l}\text { Proceso } \\
\text { Corto Anc } \\
\text { (A-P) Api }\end{array}$ & $\begin{array}{l}\text { Proceso } \\
\text { Corto Gro } \\
\text { (M-L) Api }\end{array}$ & $\begin{array}{l}\text { Proceso } \\
\text { corto Anc } \\
\text { (A-P) Prox }\end{array}$ & $\begin{array}{l}\text { Proceso } \\
\text { corto Gro } \\
\text { (M-L) Prox }\end{array}$ & $\begin{array}{l}\text { Proceso } \\
\text { largo Anc } \\
\text { (A-P) Api }\end{array}$ & $\begin{array}{l}\text { Proceso } \\
\text { largo Gro } \\
\text { (M-L) Api }\end{array}$ & $\begin{array}{l}\text { Proceso } \\
\text { largo Anc } \\
\text { (A-P) Prox }\end{array}$ \\
\hline X & 1,22 & 0,82 & 1,99 & 1,27 & 0,78 & 0,77 & 1,25 \\
DE & 0,13 & 0,11 & 0,25 & 0,10 & 0,04 & 0,11 & 0,24 \\
Rango & $0,9-1,46$ & $0,48-1$ & $1,49-2,42$ & $1,04-1,47$ & $0,71-0,84$ & $0,65-1,07$ & $0,93-1,71$ \\
\hline
\end{tabular}

Proceso Corto Anc (A-P) Api: Ancho apical (antero-posterior) de proceso corto (1 en Fig. 2).

Proceso Corto Gro (M-L) Api: Grosor apical (medio-lateral) de proceso corto (2 en Fig. 2).

Proceso Corto Anc (A-P) Prox: Ancho proximal (antero-posterior) de proceso corto (3 en Fig. 2).

Proceso Corto Gro (M-L) Prox: Grosor proximal (medio-lateral) de proceso corto (4 en Fig. 2).

Proceso Lar Anc (A-P) Api: Ancho apical (antero-posterior) de proceso largo (5 en Fig. 2).

Proceso Lar Gro (M-L) Api: Grosor apical (medio-lateral) de proceso largo (6 en Fig. 2).

Proceso Lar Anc (A-P) Prox: Ancho proximal (antero-posterior) de proceso largo (7 en Fig. 2). 


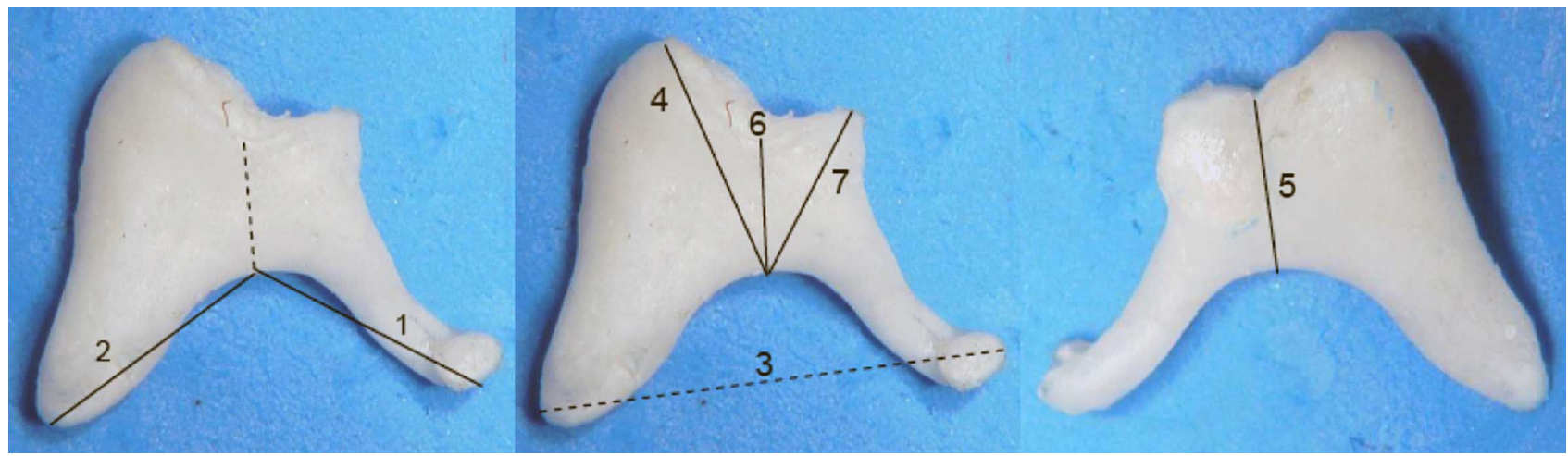

Fig. 1. Medidas del yunque en cuerpo, proceso corto y proceso largo.

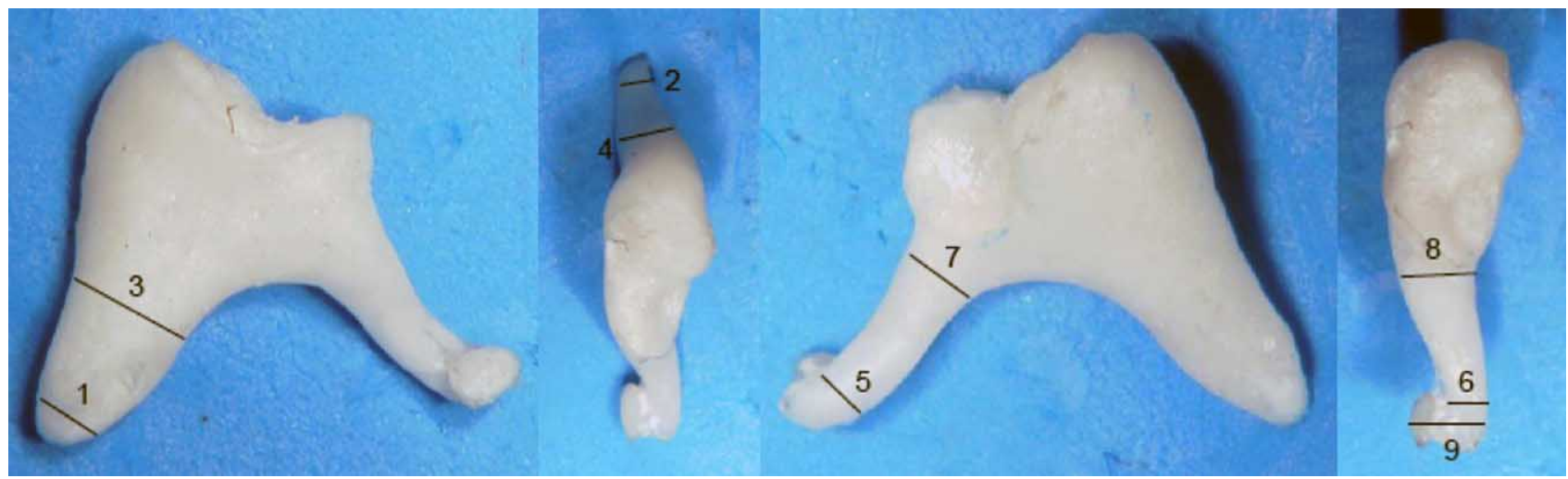

Fig. 2. Medidas del grosor y ancho proximal y apical de los procesos corto y largo del yunque

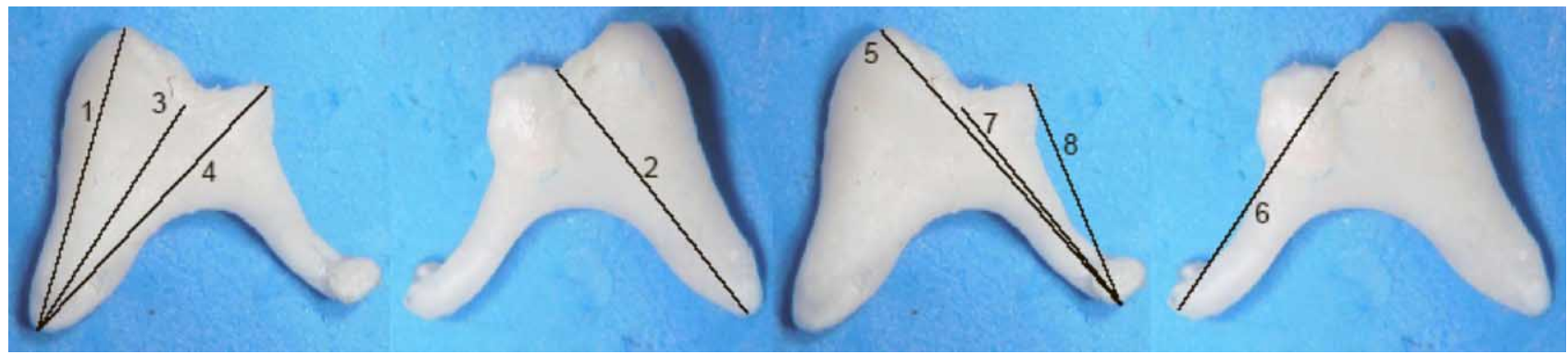

Fig. 3. Medidas de la meseta y carilla articular de la articulación incudo-maleolar.

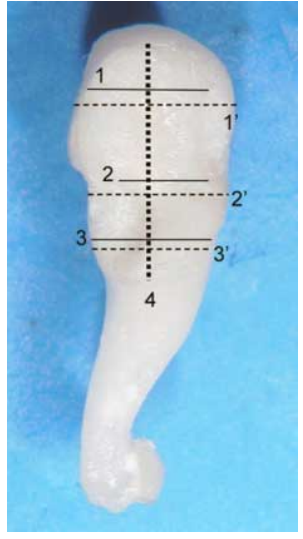

Fig. 4. Medidas de longitudes apico-coronales del yunque en zonas medial y lateral.

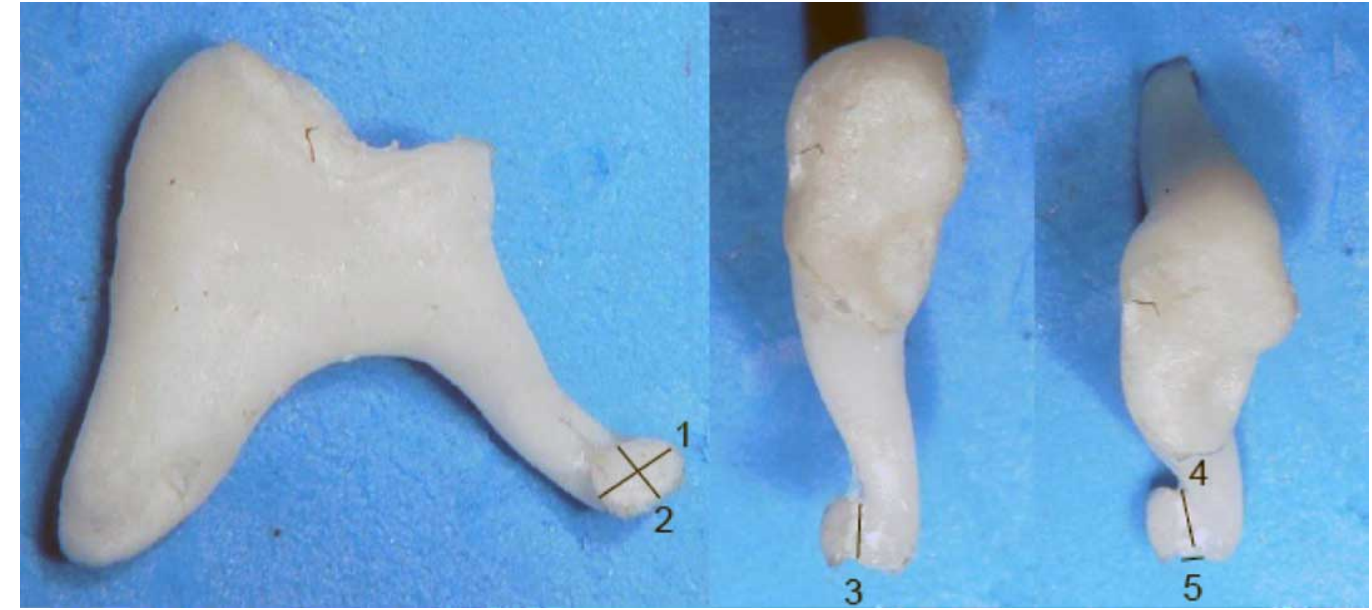

Fig. 5. Medidas del proceso lenticular y cuello del proceso. 
Tabla III. Medidas del grosor y ancho proximal y apical (incluida proceso lenticular) de proceso largo del yunque y medidas de la meseta y carilla articular de la articulación incudo-maleolar (ver Fig. 2 y 3).

\begin{tabular}{lllllllll}
\hline Medida & $\begin{array}{l}\text { Proceso } \\
\text { largo Gro } \\
\text { (M-L) Prox }\end{array}$ & $\begin{array}{l}\text { Proceso largo } \\
\text { Gro (M-L) } \\
\text { Api-Lent }\end{array}$ & $\begin{array}{l}\text { Anc Cari- } \\
\text { P }\end{array}$ & $\begin{array}{l}\text { Anc M-L } \\
\text { Mez P }\end{array}$ & $\begin{array}{l}\text { Anc Car- } \\
\text { Med }\end{array}$ & $\begin{array}{l}\text { Anc M-L } \\
\text { Mez _ }\end{array}$ & $\begin{array}{l}\text { Anc } \\
\text { Car-A }\end{array}$ & $\begin{array}{l}\text { Anc M-L } \\
\text { Mez A }\end{array}$ \\
\hline X & 1,43 & 1,13 & 1,42 & 2,13 & 1,45 & 1,83 & 1,82 & 1,81 \\
DE & 0,17 & 0,14 & 0,27 & 0,09 & 0,20 & 0,14 & 0,14 & 0,14 \\
Rango & $0,94-1,72$ & $0,92-1,52$ & $0,81-1,8$ & $1,91-2,29$ & $0,84-1,73$ & $1,54-2,07$ & $0,93-1,71$ & $1,52-2,08$ \\
\hline
\end{tabular}

Proceso Lar Gro (M-L) Prox: Grosor proximal (medio-lateral) de proceso largo (8 en Fig. 2).

Proceso Lar Gro (M-L) Procesoi-Lent: Grosor apical (medio-lateral) desde proceso largo a carilla lenticular (9 en Fig. 2).

Anc Cari-P: Ancho de la carilla más posterior de la articulación incudo-maleolar (1 en Fig. 3).

Anc M-L Mez P: Ancho de la meseta más posterior de la articulación incudo-maleolar (1’en Fig. 3).

Anc Car-Med: Ancho de la carilla media de la articulación incudo-maleolar (2 en Fig. 3).

Anc M-L Mez 1/2: Ancho de la meseta media de la articulación incudo-maleolar (2' en Fig. 3).

Anc Car-A: Ancho de la carilla más anterior de la articulación incudo-maleolar (3 en Fig. 3).

Anc M-L Mez A: Ancho de la meseta más anterior de la articulación incudo-maleolar (3’ en Fig. 3).

Tabla IV. Medidas de la longitud postero-anterior de la carilla articular incudo-maleolar y medidas de longitudes apico-coronales del yunque en zonas medial y lateral (ver Fig. 3 y 4 ).

\begin{tabular}{llllllllll}
\hline Medida & $\begin{array}{l}\text { Long } \\
\text { Car A-P }\end{array}$ & $\begin{array}{l}\text { Proceso } \\
\text { corto-Car-P }\end{array}$ & $\begin{array}{l}\text { Proceso } \\
\text { corto } \\
\text { Car 1/2- } \\
\text { R }\end{array}$ & $\begin{array}{l}\text { Proceso } \\
\text { corto } \\
\text { Car 1/2- } \\
\text { C }\end{array}$ & $\begin{array}{l}\text { Proceso } \\
\text { corto }- \\
\text { Car-A }\end{array}$ & $\begin{array}{l}\text { Proceso } \\
\text { largo-Car-P }\end{array}$ & $\begin{array}{l}\text { Proceso } \\
\text { largo - } \\
\text { Car 1/2-R }\end{array}$ & $\begin{array}{l}\text { Proceso } \\
\text { largo - } \\
\text { Car 1/2-C }\end{array}$ & $\begin{array}{l}\text { Proceso } \\
\text { largo - } \\
\text { Car-A }\end{array}$ \\
\hline $\mathrm{X}$ & & & 4,10 & 5,49 & 6,51 & 4,99 & 3,92 & 3,92 & 4,66 \\
DE & 2,93 & 5,35 & 0,22 & 0,25 & 0,32 & 0,28 & 0,28 & 0,35 & 0,30 \\
Rango & $2,38-3,2$ & $3,94-4,72$ & $3,61-4,42$ & $4,95-5,91$ & $5,66-7,05$ & $4,59-5,54$ & $4,59-5,54$ & $3,34-4,63$ & $4,23-5,32$ \\
\hline
\end{tabular}

Long Car A-P: Longitud antero-posterior de la carilla de la articulación incudo-maleolar (4 en Fig. 3).

Proceso Corto-Car-P: Longitud desde ápice de proceso corto a carilla incudo-maleolar posterior (1 en Fig. 4).

Proceso Corto Car 1/2-R: Longitud desde ápice de proceso corto a carilla incudo-maleolar media rostral (lateral) (2 en Fig. 4).

Proceso Corto Car 1/2-C: Longitud desde ápice de proceso corto a carilla incudo-maleolar media caudal (medial) (3 en Fig. 4).

Proceso Corto-Car-A: Longitud desde ápice de proceso corto a carilla incudo-maleolar anterior (4 en Fig. 4).

Proceso Largo-Car-P: Longitud desde ápice de proceso largo a carilla incudo-maleolar posterior (5 en Fig. 20).

Proceso Largo - Car 1/2-R: Longitud desde ápice de proceso largo a carilla incudo-maleolar media rostral (lateral) (6 en Fig. 4).

Proceso Largo - Car 1/2-C: Longitud desde ápice de proceso largo a carilla incudo-maleolar media caudal (medial) (7 en Fig. 4)

Proceso Largo-Car-A: Longitud desde ápice de proceso largo a carilla incudo-maleolar anterior (8 en Fig. 4).

Tabla V. Medidas de proceso lenticular y cuello de proceso (ver Fig. 5).

\begin{tabular}{llllll}
\hline Medida & Lentic A-P & Lentic R-C & $\begin{array}{l}\text { Cuello } \\
\text { Lent A-P }\end{array}$ & $\begin{array}{l}\text { Cuello } \\
\text { Lent R-C }\end{array}$ & $\begin{array}{l}\text { Gro Plat + } \\
\text { Cuello }\end{array}$ \\
\hline X & 0,81 & 0,56 & 0,68 & 0,47 & 0,36 \\
DE & 0,10 & 0,09 & 0,13 & 0,10 & 0,16 \\
Rango & $0,64-1,02$ & $0,44-0,73$ & $0,4-0,9$ & $0,36-0,72$ & $0,14-0,87$
\end{tabular}

Lentic A-P: Longitud antero-posterior de carilla lenticular (1 en Fig. 5).

Lentic R-C: Longitud rostro-caudal de carilla lenticular (2 en Fig. 5).

Cuello Lent A-P: Longitud antero-posterior de cuello de carilla lenticular (3 en Fig. 5).

Cuello Lent R-C: Longitud rostro-caudal de cuello de carilla lenticular (4 en Fig. 5).

Gro Plat + Cuello: Grosor de cuello de carilla lenticular (5 en Fig. 5).

Tabla VI. Medidas pareadas del yunque en tres registros que mostraron diferencias.

\begin{tabular}{lllllll}
\hline & Izquierdo & Derecho & Diferencia & IC 95\% & & t Student pareada \\
\cline { 5 - 7 } & & & & Lim. inf & Lim. Sup. & valor p \\
\hline Anc Cari-PI & $1,52 \pm 0,22$ & $1,34 \pm 0,30$ & $0,17 \pm 0,26$ & 0,00 & 0,35 & $\mathbf{0 , 0 4 7 2}$ \\
Anc Car-Med & $1,56 \pm 0,13$ & $1,33 \pm 0,21$ & $0,23 \pm 0,14$ & 0,13 & 0,33 & $\mathbf{0 , 0 0 0 3}$ \\
Gro Plat +Cuello & $0,38 \pm 0,13$ & $0,30 \pm 0,10$ & $0,08 \pm 0,11$ & 0,01 & 0,15 & $\mathbf{0 , 0 3 7 6}$ \\
\hline
\end{tabular}

Anc Cari-P: Ancho de la carilla más posterior de la articulación incudo-maleolar (Lado Izquierdo).

Anc Car-Med: Ancho de la carilla media de la articulación incudo-maleolar (Lado Izquierdo).

Gro Plat + Cuello: Grosor de cuello de carilla lenticular (Lado Izquierdo). 


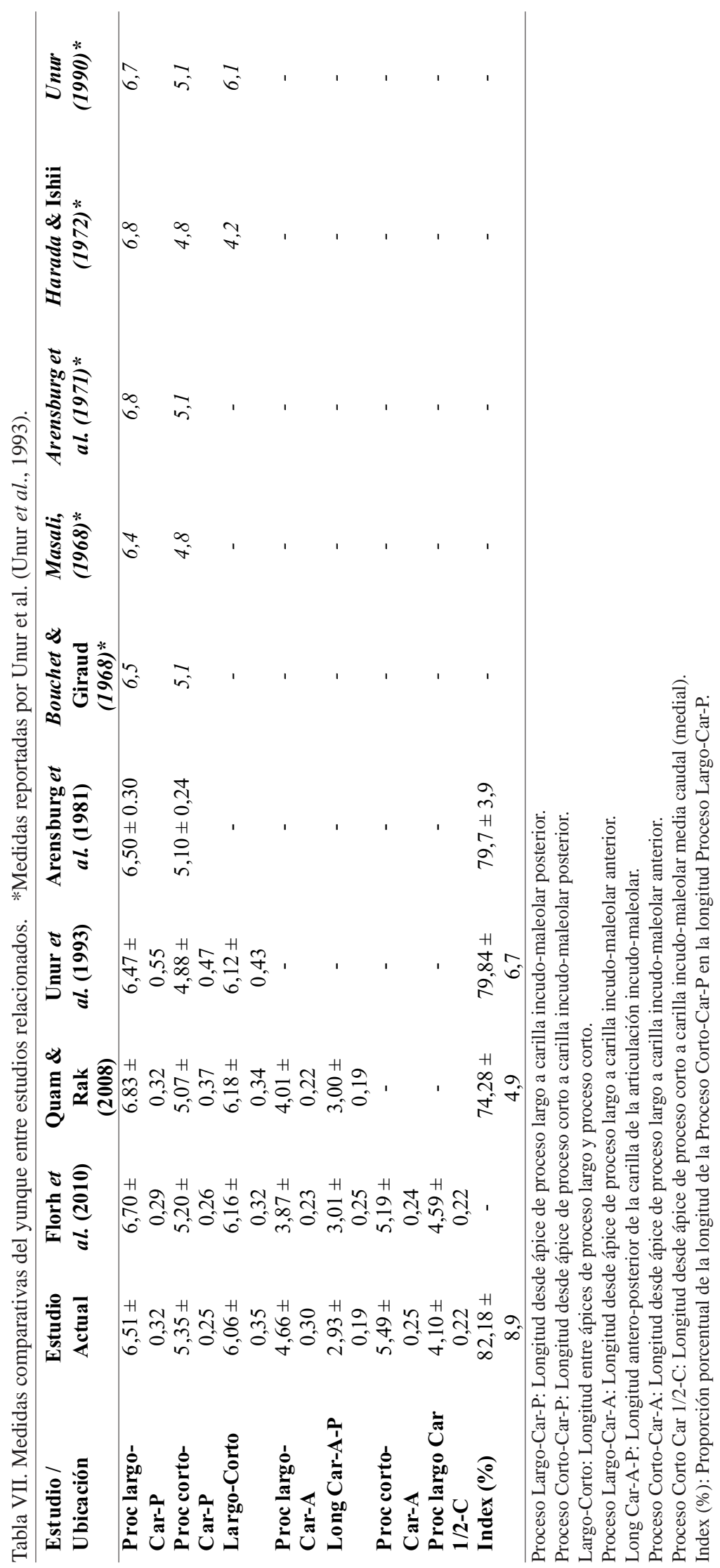

\section{DISCUSIÓN}

Se han estudiado tanto la estructura como la función de los huesecillos del oído medio en humanos (Bruintje, 1990; Anson \& Donaldson, 1991). Igualmente la dimensión y el peso de estos comparados entre fetos, adultos y niños que ha mostrado una sutil variación por crecimiento después del nacimiento (Olsewski, 1989; Olsewski, 1990). Los patrones de transmisión de fuerzas y su mecánica han sido también estudiados con modelos matemáticos que reconstruyen las formas particulares de los huesecillos para su posterior procesamiento a través de estudios de elemento finito (Arensburg \& Nathan, 1971; Tonndorf \& Khanna, 1972; Sarrat et al., 1988; Sun et al., 2002; Morris et al., 2004).

De las medidas registradas en este estudio, que corresponden a treinta y cinco registros, solo pueden ser comparadas con estudios previos (Arensburg et al., 1981; Unur et al., 1993; Quam \& Rak, 2008; Flohr et al., 2010), algunas pocas medidas que corresponden en la mayoría al largo total yunque (medida en este estudio como Proceso Largo-Car-P), ancho total del yunque (medida en este estudio como Proceso Corto-Car-P), distancia entre extremos de los procesos corto y largo del yunque (medida en este estudio como Largo-Corto). Adicionalmente en el estudio de Quam \& Rak y Flohr et al., se registran la longitud del proceso largo del yunque (medida en este estudio como Proceso Largo-Car-A), y la longitud anteroposterior de la carilla articular del yunque (medida en éste estudio como Long CarA-P). El estudio de Flohr et al. es el que más registros tiene de todos los que permiten la comparación, superando a los anteriores en dos medidas adicionales: la longitud desde el ápice del proceso corto a la cresta anterior de la carilla articular de la articulación incudo-maleolar (medida en este estudio como Proceso Corto-Car-A) y la longitud desde el ápice del proceso corto a la carilla incudo-maleolar media caudal (medida en éste estudio como Proceso Corto Car 1/2-C). Todas las comparaciones de estos registros están en la Tabla VII. 
Nuestros resultados coinciden con la percepción general de que el yunque es un hueso estable sin presencia de importantes variaciones (Unur et al.), incluidos los cambios postnatales (Olsewski, 1989; Olsewski, 1990). Arensburg et al. encontraron los oscículos con morfometría muy estables en poblaciones históricas y prehistóricas con contrastes étnicos, temporales (miles de años) y geográficos amplios. Crevecoeur (2007) estudió la morfometría y la relación filogenética del homínido hombre de diferentes épocas evolutivas midiendo los oscículos desde antes del Neandertal, pasando por el paleolítico y llegando hasta el humano moderno, concluyendo que estos huesecillos son morfométricamente muy estables tanto en poblaciones pre-históricas como históricas, como aquellas étnica y geográficamente diferentes. Este autor matiza que el tamaño del cráneo y el cuerpo no guardan correlación con el de los oscículos, lo que implica valor filogenético y se entiende como pocas variaciones anatómicas para estas estructuras óseas. Quam \& Rak no encontraron diferencias morfológicas significativas salvo algunas diferencias morfométricas sutiles entre oscículos de hombre moderno y Neandertal que asocian a la evolución. La Tabla VII per- mite observar estas sutiles variaciones con otras épocas del género Homo (Arensburg et al.; Unur et al.; Quam \& Rak; Flohr et al.).

El conjunto de medidas morfométricas recolectadas del yunque en especímenes colombianos amplia la base de datos existente e igualmente es información provechosa que estudie el funcionamiento de los oscículos mediante la construcción de modelos virtuales que permitan comprender la mecánica de la transmisión de la energía sonora (Willi et al., 2002). El registro de las medidas buscó corroborar lo encontrado en otras investigaciones. De esta manera se amplía la información desde una perspectiva étnica y geográfica diferente ya que no hay trabajos parecidos en población mestiza Colombiana. Finalmente un análisis pareado de estas medidas (Tabla VI) encontró que hubo tres diferencias de lado en los registros que consideramos sugestivas debido a la homogeneidad presentada en los otros treinta y dos registros.

En conclusión, encontramos que la morfología del yunque es homogénea y estable.

RAMIREZ, L. M. \& BALLESTEROS, L. E. Anthropometry of the incus and incudomalleolar and incudostapedial joints in humans: A direct anatomical study. Int. J. Morphol., 31(2):539-545, 2013.

SUMMARY: Current studies on anatomical variations of the incus in humans from the morphometric register are insufficient. Structural and dimensional characteristics of the incus are registered in 23 samples of Colombian adult mestizo population. Registered measures involved incus body, incudo-maleolar joint, long process, short process and incudo-stapedial joint. Only eight of the thirty-five records taken in this study could be thoroughly compared to other anterior studies, which showed similarities. The total length of the anvil was 6,51 $\mathrm{mm}$ (SD 0.32), the length of the long process of the incus was $5.35 \mathrm{~mm}$ (SD 0.25), the distance between processes long and short was $6.06 \mathrm{~mm}$ (SD 0.35) and the ratio of lengths between both processes was $82.18 \%$ (SD 8.9\%). In accordance with the studied population we found paired variation in the anatomy of the incus in only three of the records taken, however, we believe that it is a uniform and morphometrically stable bone.

KEY WORDS: Oscicles; Incus; Incudo-malleolar joint; Long process; Short process; Incudo-stapedial joint.

\section{REFERENCIAS BIBLIOGRÁFICAS}

Anson, B. J. \& Donaldson, J. A. Surgical anatomy of the temporal bone. $3^{\text {a }}$ ed. Philadelphia, WB Saunders, 1991.

Arensburg, B.; Harell, M. \& Nathan, H. The human middle ear ossicles, morphometry and taxonomic implications. J. Hum. Evol., 10(2):199-205,1981.

Arensburg, B. \& Nathan, H. Observations on a notch in the short (Superior or Posterior) process of the incus. Acta Anat. (Basel), 78(1):84-90, 1971

Asai, M.; Heiland, K. E.; Huber, A. M. \& Goode, R. L. Evaluation of cement incus replacement prosthesis in a temporal bone model. Acta Otolaryngol., 119(5):573-6, 1999.

aWengen, D. F.; Nishihara, S., Kurokawa, H. \& Goode, R. L. Measurements of the stapes superstructure. Ann. Otol. Rhinol. Laryngol., 104(4 Pt. 1):311-6, 1995.

Bouchet, A. \& Giraud, M. Contrubituon a l'etude morphologique et radiologique des osselets de l'ouie. Compte rendu de l' Association des Anatomists 53 Congrés, 141:588-600, 1968.

Bruintje, Tj. D. The auditory ossicles in human skeletal remains from a leper cemetery in Chichester, England. J. Archaeol. Sci., 17(6):627-33, 1990. 
Cole, F. G. In History of comparative anatomy. London, Macmillan \& Co. Ltd., 1949. pp.69-120.

Crevecoeur, I. New discovery of an Upper Paleolithic auditory ossicle: the right malleus of Nazlet Khater 2. J. Hum. Evol., 52(3):341-5, 2007.

Farahani, R. M. \& Nooranipour, M. Anatomy and anthropometry of human stapes. Am. J. Otolaryngol., 29(1):42-7, 2008.

Flohr, S.; Leckelt, J.; Kierdorf, U. \& Kierdorf, H. How reproducibly can human ear ossicles be measured? A study of inter-observer error. Anat. Rec. (Haboken), 293(12):2094-106, 2010.

Guyton, A. C. \& Hall, J. E. Tratado de Fisiología Médica. 11ª ed. Barcelona, Elsevier, 2007.

Harada, O. \& Ishii, H. The condition of the auditory ossicles in microtia: findings in 57 middle ear operations. Plast. Reconstr. Surg., 50(1):48-53, 1972.

Mills, R. P. Ossicular geometry and the choice of technique for ossiculoplasty. Clin. Otolaryngol. Allied Sci., 16(5):476-9, 1991.

Morris, D. P.; Bance, M.; van Wijhe, R. G.; Kiefte, M. \& Smith, R. Optimum tension for partial ossicular replacement prosthesis reconstruction in the human middle ear. Laryngoscope, 114(2):305-8, 2004.

Murugasu, E.; Puria, S. \& Roberson, J. B. Malleus-to-footplate versus malleus-to-stapes-head ossicular reconstruction prostheses: temporal bone pressure gain measurements and clinical audiological data. Otol. Neurotol., 26(4):572-82, 2005.

Nishihara, S. \& Goode, R. L. Experimental study of the acoustic properties of incus replacement prostheses in a human temporal bone model. Am. J. Otol., 15(4):485-94, 1994.

Nishizaki, K. \& Anniko, M. Developmental morphology of the middle ear. Auris Nasus Larynx, 24(1):31-8, 1997.

Olsewski, J. Structure of the middle ear in infants. Otolaryngol. Pol., 43(4):278-83, 1989.

Olsewski, J. The morphometry of the ear ossicles in humans during development. Anat. Anz., 171(3):187-91, 1990.

Quam, R. \& Rak, Y. Auditory ossicles from southwest Asian Mousterian sites. J. Hum. Evol., 54(3):414-33, 2008.

Ramirez, L. M. \& Ballesteros, L. E. Human Oscicular Chain Articulations: Asymmetric Sound Transmission. Int. J. Morphol., 28(4):1059-68, 2010.

Sarrat, R.; García Guzman, A. \& Torres, A. Morphological variations of Human ossicula tympani. Acta Anat. (Basel), 131(2):146-9, 1988
Sun, Q.; Gan, R. Z.; Chang, K. H. \& Dormer, K. J. Computerintegrated finite element modeling of human middle ear. Biomech. Model. Mechanobiol., 1(2):109-22, 2002.

Tonndorf, J. \& Khanna, S. M. Tympanic-membrane vibrations in human cadaver ears studied by time-averaged holography. $J$. Acoust. Soc. Am., 52(4):1221-33, 1972.

Unur, E.; Aycan, K. \& Ekinci, N. The study of incus from morphometric view. Erciyes Medical J., 15:16-9, 1993.

Willi, U. B.; Ferrazzini, M. A. \& Huber, A. M. The incudo-malleolar joint and sound transmission losses. Hear Res., 174(1-2):3244, 2002.

Whyte Orozco, J.; Cisneros Gimeno, A. I.; Urieta Carpi, J. J.; Yus Gotor, C.; Gañet Solé, J.; Torres del Puerto, A.; et al. Ontogenic peculiarities of the human tympanic ossicular chain. Acta Otorrinolaringol. Esp., 54(1):1-10, 2003.

Whyte, J. R.; González, L.; Cisneros, A. I.; Yus, C.; Torres, A. \& Sarrat, R. Fetal development of the human tympanic ossicular chain articulations. Cells Tissues Organs, 171(4):241-9, 2002.

\author{
Dirección para Correspondencia: \\ Luis Miguel Ramirez Aristeguieta, DDS, MS, MSc \\ Profesor Asociado Facultad de Odontologia \\ Universidad de Antioquia \\ Grupo de Investigación Estomatologia Biomedica \\ Medellin \\ COLOMBIA
}

Tel: (57) 3157523178

Email: Imra3@yahoo.com

Recibido : 20-02-2012

Aceptado: 17-11-2012 